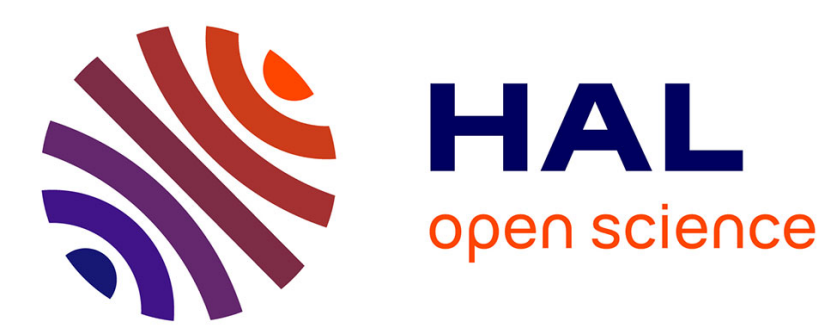

\title{
Identification photométrique des particules s'arretant dans les chambres à bulles
}

\author{
E. Hourani, T. Yuasa
}

\section{To cite this version:}

E. Hourani, T. Yuasa. Identification photométrique des particules s'arretant dans les chambres à bulles. Revue de Physique Appliquée, 1966, 1 (3), pp.252-256. 10.1051/rphysap:0196600103025200 . jpa-00242729

\section{HAL Id: jpa-00242729 https://hal.science/jpa-00242729}

Submitted on 1 Jan 1966

HAL is a multi-disciplinary open access archive for the deposit and dissemination of scientific research documents, whether they are published or not. The documents may come from teaching and research institutions in France or abroad, or from public or private research centers.
L'archive ouverte pluridisciplinaire HAL, est destinée au dépôt et à la diffusion de documents scientifiques de niveau recherche, publiés ou non, émanant des établissements d'enseignement et de recherche français ou étrangers, des laboratoires publics ou privés. 


\title{
IDENTIFICATION PHOTOMÉTRIQUE DES PARTICULES S'ARRETANT DANS LES CHAMBRES A BULLES
}

\author{
Par E. HOURANI et T. YUASA, \\ Institut de Physique Nucléaire, Orsay, France.
}

\begin{abstract}
Résumé. - On décrit la mise au point d'un microdensitomètre et une méthode photométrique, destinées à l'identification des particules s'arrêtant dans les chambres à bulles et donnant sur les clichés des trajectoires à traînées continues de bulles. On s'est intéressé aux protons et aux particules $\alpha$ de la réaction ${ }^{12} \mathrm{C}(\mathrm{p}, \mathrm{p} \alpha){ }^{8} \mathrm{Be}$ provoquée dans une chambre à bulles à propane par les protons du synchrocyclotron d'Orsay. L'identification a été possible dans les clichés ayant, pour les protons incidents, une faible densité de bulles apparente.
\end{abstract}

Abstract. - The combination of a microdensitometer and a photometric method used to identify the particles stopping in a bubble chamber when their tracks are continuous on film, is described. The protons and the $\alpha$-particles from the ${ }^{12} \mathrm{C}(\mathrm{p}, \mathrm{p} \alpha)^{8} \mathrm{Be}$ reaction induced in a propane bubble chamber by a proton beam of the Orsay synchrocyclotron are studied. The identification is possible in the films when the incident protons have low apparent bubble density.

1. Introduction. - L'analyse cinématique des réactions nucléaires, d'après les clichés de chambre à bulles et les émulsions nucléaires, ne donne pas toujours une solution unique. On a recours alors à des critères auxiliaires, en particulier à la densité de bulles ou de grains sur les trajectoires [1]-[9]; mais ce critère n'est pas valable pour les particules s'arrêtant dans la chambre à bulles où les émulsions nucléaires après un petit parcours dont les trajectoires observées se présentent en traînées continues de bulles ou de grains; or, dans les émulsions nucléaires, on a pu tirer, à partir des caractéristiques photométriques des traînées continues de grains, des informations sur la nature des particules créatrices ([10] à [14]). Nous nous sommes donc proposés d'appliquer une méthode photométrique pour les traînées de bulles. Sur les clichés d'une chambre à bulles à propane [15], nous avons essayé de distinguer entre les protons et les particules $\alpha$ concernant la réaction ${ }^{12} \mathrm{C}(\mathrm{p}, \mathrm{p} \alpha)^{8} \mathrm{Be}$ provoquée par les protons du synchrocyclotron d'Orsay [16].

2. Dispositif expérimental. - A) Microdensiтомѐтre. - Pour l'étude photométrique d'une trajectoire dans un cliché, nous avons mis au point un microdensitomètre qui comprend un système de projection et un microphotomètre. Le cliché est projeté par un objectif sur une table. Le microphotomètre comporte une fente définie dans une boîte appelée tête du microphotomètre (fig. 1) posée sur la table et un conduit de lumière ${ }^{1}$ ) qui guide la lumière entrée dans la fente à la photocathode d'un photomultiplicateur. Nous lisons la tensions aux

(1) Constitué de fibres de verre juxtaposées; une fibre consiste en une partie centrale recouverte d'une couche d'indice inférieur. bornes de la résistance de charge du P. M. à l'aide d'un voltmètre numérique. Notons qu'il y a deux vis micrométriques donnant à la fente des déplacements précis dans deux sens perpendiculaires.

En fonctionnement normal, d'une part la lecture $\mathrm{du}$ voltmètre est proportionnelle au flux lumineux entré dans la fente et d'autre part la distribution d'intensité lumineuse, relevée par la fente, sur l'image de la plage à mesurer du cliché n'est pas gênée par un effet de diffusion de lumière parasite, [17]. Pour obtenir ce fonctionnement normal, il a fallu d'une part travailler dans la région linéaire du P. M. et d'autre part mettre un diaphragme à la plage à mesurer. On teste le fonctionnement en mesurant par l'expression $\log _{10}\left(V_{0} / V\right)$ la densité optique $d$ connue de filtres étalonués, $V$ et $V_{0}$ étant les lectures du voltmètre en plaçant ou non le filtre à l'endroit du cliché (rappelons que si un filtre transmet d'un flux lumineux $\Phi_{0}$ une partie $\Phi$ sa densité optique $d$ est telle que $\left.d=\log _{10}\left(\Phi_{0} / \Phi\right)\right)$.

Les fluctuations sur les flux lumineux sont inférieures à $1 \%$ et la reproductibilité de mesure des densités optiques est à $\pm 2 \%$.

B) Clichés de chambres a bulles. - Les trajectoires que nous analysons sur clichés représentent les traces (laissées par la particule lors de son passage dans la chambre à bulles) au sortir de trois étapes successives : formation de bulles par ces traces, illumination des bulles, puis photographie. Nous allons dire un mot du rôle représentatif de chaque étape.

a) Fornıation de bulles : On a établi expérimentalement, en comptant les bulles et en étudiant la répartition des lacunes entre les bulles, que la densité de bulles varie le long d'une trajectoire 

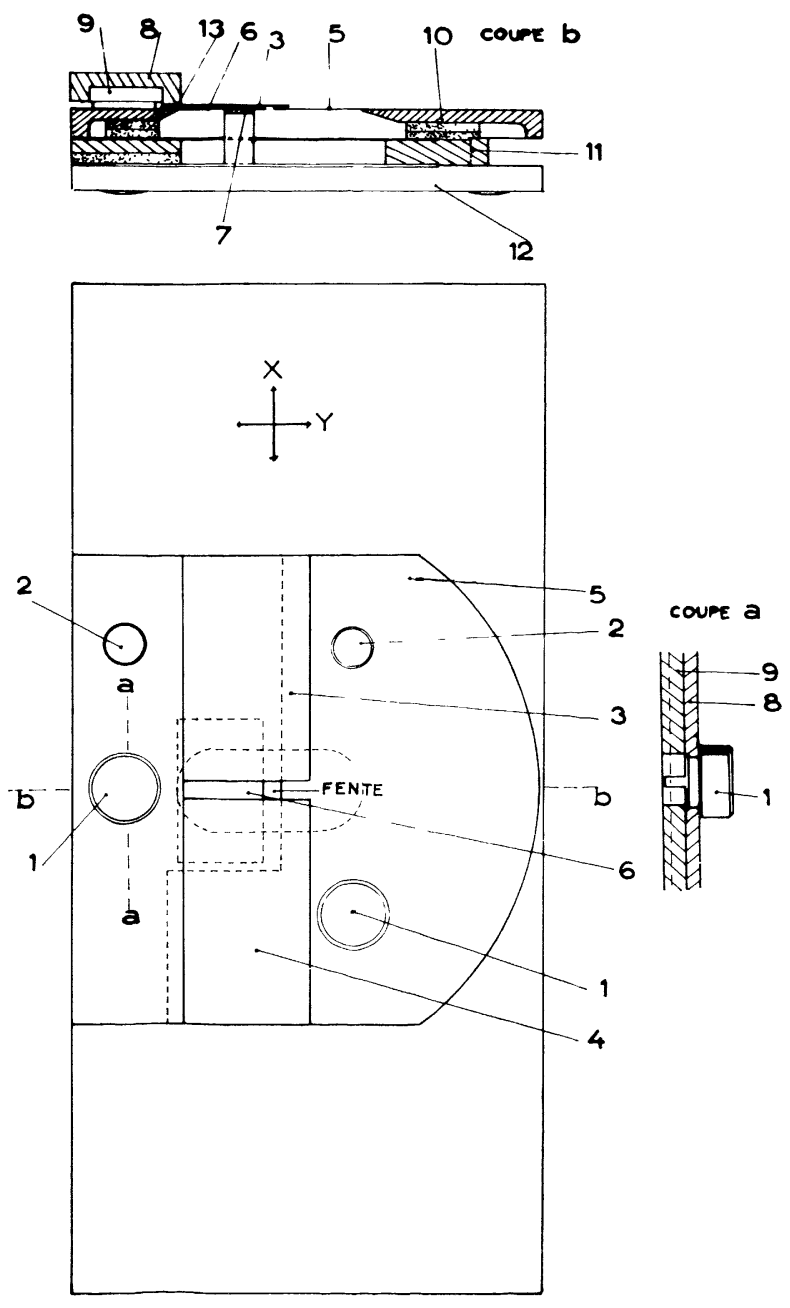

Fig. 1. - Tête du microphotomètre.

1. Cale de réglage. -2 . Vis de blocage. - 3. Plaque mobile des X. - 4. Plaque fixe des X. - 5. Plaque mobile des Y. - 6. Plaque fixe des Y. - 7. Étrier. - 8. Coulisse. - 9. Glissière. - 10. Plaque support de 5. - 11. Plaque fixe. - 12. Plaque de base. 13. Cale.

comme $1 / \beta^{2}, \beta$ étant la vitesse de la particule en unité $c[1]-[9]$. Se basant sur le fait que cette loi en $1 / \beta^{2}$ est la conséquence de la formation des bulles par les rayons $\delta$ de quelques centaines d'eV [1], [3], [18], [19], un calcul permet de généraliser cette loi pour des particules de charge $Z$; la densité de bulles $g$ calculée est telle que [3], [18] :

$$
g=g_{0} Z^{2} / \beta^{2}
$$

$g_{0}$ dépend de l'état du milieu liquide de la chambre à bulles.

Notons que la loi $g=g_{0} Z^{2} / \beta^{2}$ est établie pour des particules d'énergie suffisamment élevée pour que les bulles soient distinctes sur les trajectoires. Elle n'est pas alors directement valable pour le cas qui nous intéresse où les particules sont de très basse énergie et donnent sur clichés des trajectoires en traînées continues de bulles. Nous admettrons cependant que la loi $g={ }^{-} Z_{0} / \beta^{2}$ est valable pour les particules de très basse énergie quand les bulles sont, au cours de grossissement, assez petites pour qu'elles soient distinctes; les problèmes d'étroit voisinage des bulles, tels que l'absorption mutuelle, sont négligeables.

b) Illumination: L'éclairage dans la chambre à bulles n'est pas uniforme. De plus, une trajectoire dans la chambre à bulles peut avoir des orientations différentes. Donc un point d'une trajectoire va avoir sa brillance vers la caméra dépendant de sa position et de l'orientation de la trajectoire, et par conséquent, les trajectoires sur film ne sont pas directement comparables.

c) Photographie : La densité optique en un point d'une trajectoire sur film développé de chambres à bulles est liée à la brillance du point homologue de la trajectoire photographiée par la courbe caractéristique du film utilisé. Nous avons établi cette courbe dans les mêmes conditions d'utilisation du film. Les flux lumineux d'irradiation ont été variés avec $_{i a}^{k} l$ 'interposition de filtres neutres étalonnés.

3. Méthode d'analyse. - Considérons les bulles immédiatement eprès leur formation quand elles sont assez petites pour qu'étant très voisines elles ne s'absorbent pas. Si on éclaire une portion de trajectoire, il y aura de la lumière diffusée dont l'intensité est proportionnelle à la densité de bulles en cette portion. Pour avoir cette intensité, il suffit d'établir la courbe de variation de la brillance transversalement à la portion; l'intensité est l'aire, I, de cette courbe.

Nous photographions les trajectoires lorsque les bulles ont grossi et qu'alors des bulles très voisines se sont absorbées. A cause de cette absorption, l'aire $I$ de la courbe de brillance établie dans un balayage transversal d'une portion de trajectoire n'est plus proportionnelle à la densité de bulles préalablement formées. Nous continuons toutefois à caractériser la portion de trajectoire par l'aire $I$. C'est la grandeur que nous mesurons.

Pour établir $I$, nous effectuons par une fente fine du microdensitomètre un balayage transversal de l'image projetée de la trajectoire. Les dimensions de la fente doivent être petites par rapport à celles de la trajectoire pour qu'elle puisse résoudre ses détails photométriques. La largeur de l'image de la trajectoire sur la table de projection est d'environ $0,6 \mathrm{~mm}$; nous avons utilisé une fente de $0,15 \mathrm{~mm} \times 0,15 \mathrm{~mm}$. Le balayage de l'image de la trajectoire par la fente donne la courbe de variation des densités optiques de la portion balayée. A partir de cette courbe et de la courbe caractéristique du film, nous obtenons la courbe de brillance de la portion. L'aire $I$ de la courbe de brillance (courbe gaus- 
sienne) sera prise approximativement égale à $L \times H, L$ étant la largeur à mi-hauteur et $H$ la hauteur de cette courbe.

4. Résultats. - Nous nous sommes proposés de distinguer entre les protons et les particules $\alpha$ sur les clichés d'une chambre à bulles à propane. Nous ne disposons pas de particules $\alpha$ étalons, nous utiliserons les événements classés cinématiquement en ${ }^{12} \mathrm{C}(\mathrm{p}, \mathrm{p} \alpha)^{8} \mathrm{Be}$. Une éventuelle impureté dans ces événements n'est pas exclue. Notre méthode sera de confronter des mesures faites sur les branches pouvant être $\alpha$ avec celles sur les branches proton en vue de trouver une différence. Aussi utiliserons-nous comme protons sûrs les branches d'événements classés sans ambiguïté en ${ }^{1} \mathrm{H}(\mathrm{p}, \mathrm{p}){ }^{\mathbf{1}} \mathrm{H}$. Deux séries de mesure ont été faites.

A) Comparaison dans la même condition D'Éclairage. - Nous comparons entre les branches d'un événement ${ }^{12} \mathrm{C}(\mathrm{p}, \mathrm{p} \alpha)^{8} \mathrm{Be}$ ou ${ }^{1} \mathrm{H}(\mathrm{p}, \mathrm{p})^{1} \mathrm{H}$ au voisinage du point de fourche B (fig. $2 a)$. Au voisi-

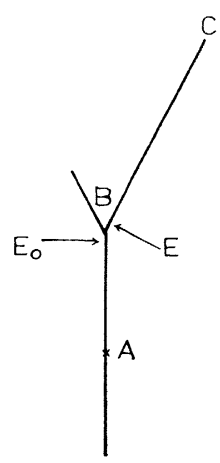

(a)

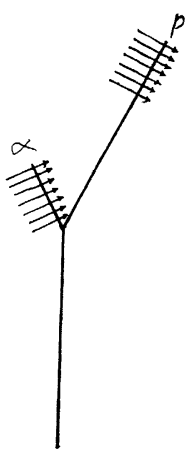

(b)
FIG. $2 a$.

nage, les branches sont dans les mêmes conditions d'éclairage; le rapport $I_{\mathrm{E}} / I_{\mathrm{E}_{0}}$ des valeurs $I$ établi

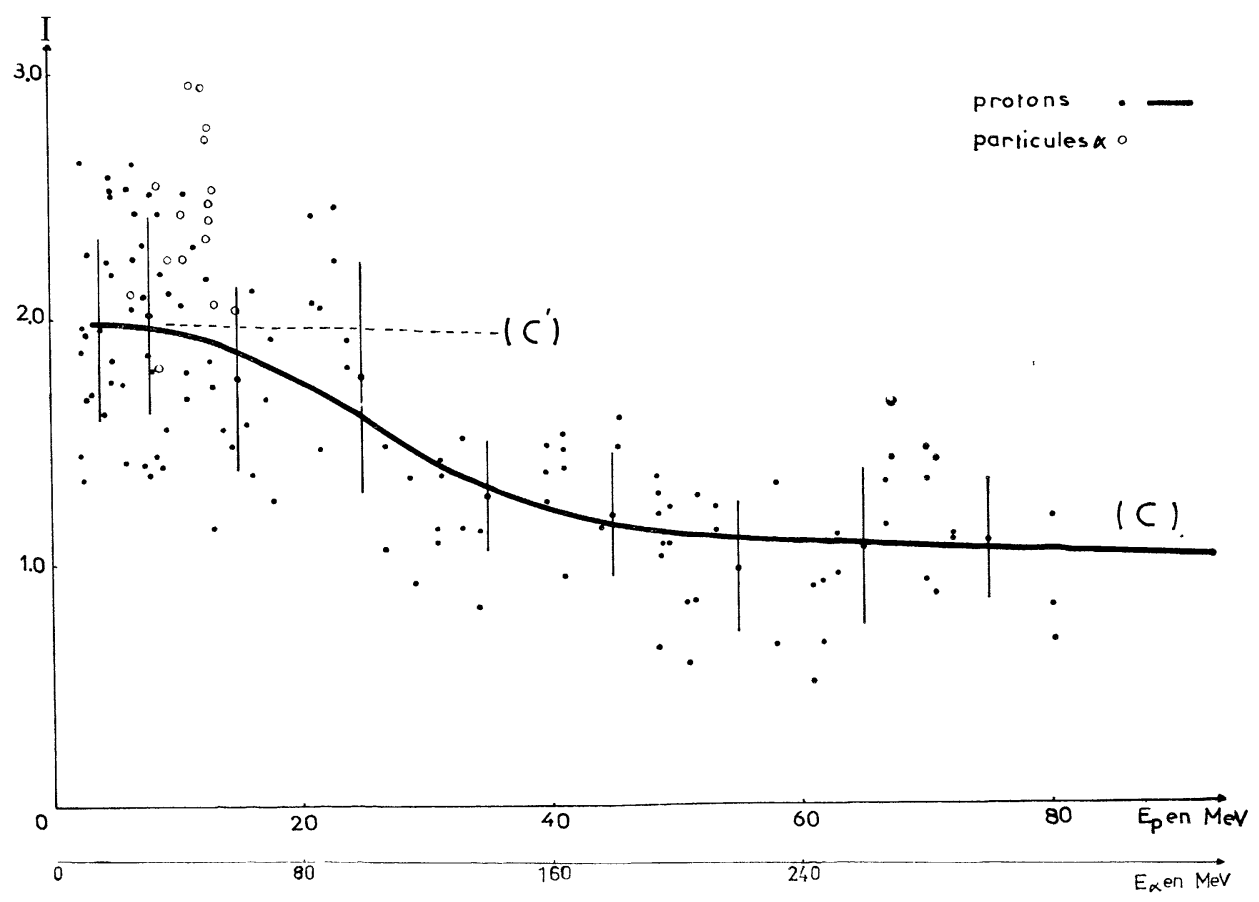

FIG. 3.

aux énergies $E$ et $E_{0}$ (fig. $2 a$ ) dépendra seulement de ces énergies $\left({ }^{2}\right)$.

Dans (fig. 3) $E_{0}=\overline{9} 3 \mathrm{MeV}$ et $I_{\mathbf{E}_{0}}$ a été normalisé à 1 ; les points représentent des mesures pour des protons de différentes énergies $E_{\mathbf{p}},(\mathrm{C})$ est la courbe

(2) $I_{\mathrm{E}}$ et $I_{\mathrm{E}_{0}}$ ont été établis dans le balayage par la fente des branches $\mathrm{BC}$ et $\mathrm{AB}(f i g .2 a)$ de façon que la fente traverse diamétralement les bulles les plus voisines de B. moyenne de ces points; les grandes fluctuations sont dues aux variations d'un cliché à un autre de la densité de bulles pour une même énergie d'une particule $\left({ }^{3}\right)$. La tendance stationnaire de la

${ }^{(3)}$ Ces variations sont dues à des fluctuations dans les conditions de fonctionnement de pression et de température de la chambre à bulles. On estime ces variations en considérant la densité de bulles apparente à une énergie donnée des protons incidents. 
courbe $(\mathrm{C})$ vers les basses énergies correspondrait à une absorption mutuelle de bulles voisines; cette absorption finit par une saturation (on pourrait imaginer que les bulles se sont jointes au cours de grossissement pour former un petit cylindre de vapeur au sein du liquide). Les petits ronds cerrespondent à des particules $\alpha$; pour une $\alpha$ donnée, $I$ a été portée en fonction de l'énergie $E_{\mathrm{p}}$ d'un proton ayant le même parcours que cette $\alpha$. Les ronds ont la tendance de se détacher des points correspondant aux protons.

Admettant que la courbe $(\mathrm{C})$ dépend de l'énergie au moyen de $g$, g étant la densité de bulles considérées, au cours de grossissement, à un moment où elles sont distinctes et admettant que la loi $g=g_{0} Z^{2} \beta^{2}$ régit ces bulles, nous prévoyons la courbe expérimentale $\left(\mathrm{C}^{\prime}\right)$ pour $\alpha$ (fig. 3). En comparant $(C)$ et $\left(C^{\prime}\right)$, nous voyons une assez nette distinction pour des $\alpha$ d'énergie supérieure à une cen-

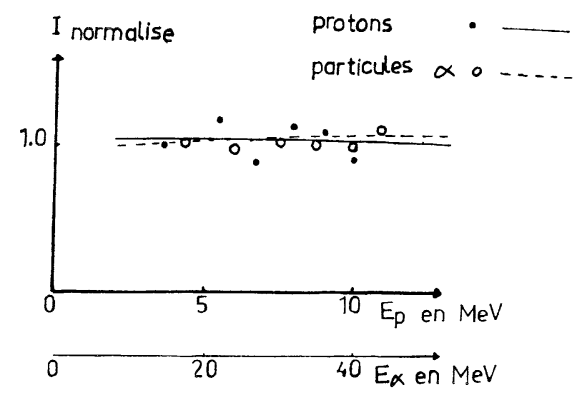

(a) taine de MeV. Pour espérer une nette distinction pour des énergies plus faibles, il faudrait atténuer les fluctuations.

B) Comparaison dans un même cliché. - Nous nous sommes proposés d'établir la grandeur $I$ dans plusieurs balayages exploitant toute la branche $\alpha$ et nous avons fait de même pour une longueur égale de la fin de la branche proton (fig. $2 b$ ). L'éclairage dans la chambre à bulles étant différent d'une branche à l'autre, nous avons normalisé pour une branche donnée les valeurs $I$ de façon à donner la valeur 1 à celle du balayage le plus voisin de la fin de cette branche.

L'intérêt de cette série de mesure est de faire la comparaison entre les deux branches d'un même événement. Ainsi les mesures comparées concernent le même cliché et sont dans les mêmes conditions de formation de bulles. Nous portons (fig. 4) l'énergie

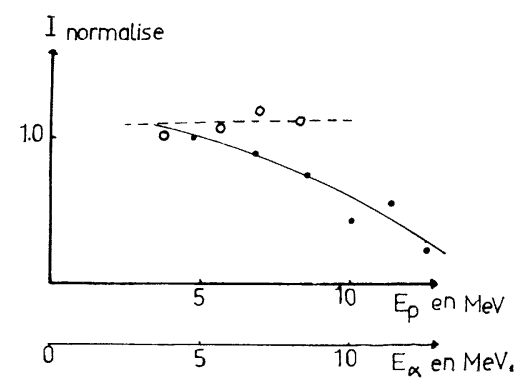

(b)

FIG. 4. - Sur la figure 4(b), en abscisses, pour les valeurs de $E_{\alpha}$ lire 0, 20, 40.

en abscisses et les $I$ normalisées en ordonnées ; nous distinguons entre deux classes d'événements :

a) des événements dont les courbes correspondant aux protons et aux particules $\alpha$ ont une allure semhlable, constante en fonction de l'énergie ( $f i g .4 a)$;

b) des événements dont la courbe correspondant à la particule $\alpha$ est constante, tandis que celle correspondant au proton décroît quand l'énergie croît (fig. 4b).

L'examen individuel des clichés analysés a montré que les clichés des événements de la classe $b$ présentent, sur les trajectoires des protons incidents, une densité de bulles nettement plus faible que la densité sur celles des événements de la classe $a$. Nous en déduisons :

- dans nos clichés à faible densité de bulles, toute la trajectoire de $\alpha$ est uniforme tandis qu'une longueur égale de la fin de trajectoire de proton ne l'est pas, et, dans nos clichés à forte densité de bulles la trajectoire de $\alpha$ et une longueur égale de la fin de celle du proton sont uniformes et semblables (fig. 5) ;

- réciproquement, parmi nos clichés seuls ceux à faible densité de bulles permettent de distinguer entre protons et particules $\alpha$.

5. Conclusion. - Par une méthode photométrique, nous avons trouvé qu'une trajectoire sur un cliché de chambre à bulles se présente pareille (saturée) en toute sa partie correspondant à des densités de bulles supérieures à un certain seuil. L'analyse photométrique situe sur une trajectoire l'emplacement de ce seuil et établit une correspondance entre la distance de l'emplacement du seuil à la fin de trajectoire et la nature des particules, rendant ainsi leur identification possible.

Nous avons pu ainsi distinguer entre les protons et les particules $\alpha$ dans nos clichés ayant, pour les protons incidents, une faible densité de bulles : les trajectoires se présentent pareilles (saturées) sur toutes leurs longueurs pour les particules $\alpha$ et à partir de l'emplacement du seuil pour les protons. Quant aux clichés à forte densité de bulles, les trajectoires s'y présentent pareilles sur toutes leurs longueurs pour les particules $\alpha$ et les protons à la fois. Quand on veut identifier photométriquement des 


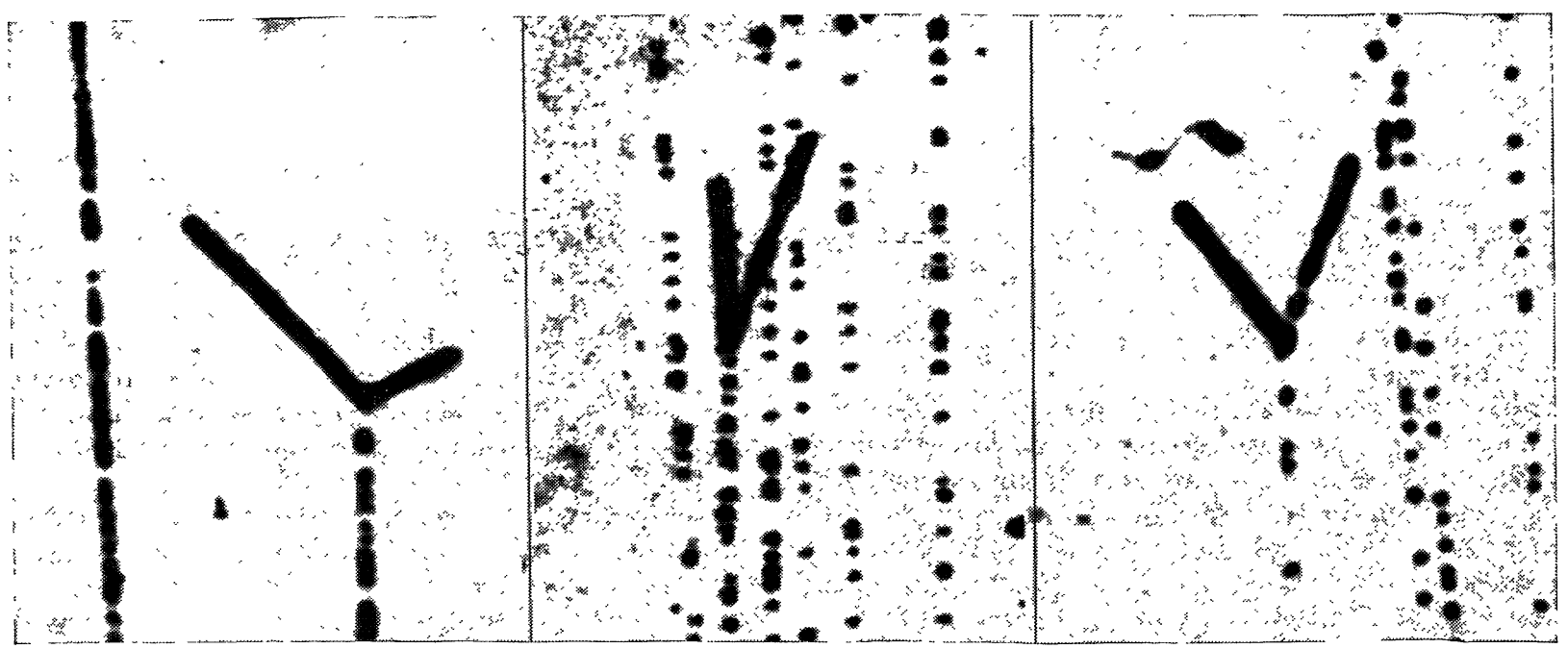

Événement non identifiable dans un cliché à forte densité de bulles.
Événement identifié ( $p, p \alpha)$ dans un cliché à assez faible densité de bulles.
Événement identifié ( $p, p \alpha)$ dans un cliché à faible densité de bulles.

Fig. 5. - Grandissement 32 par rapport aux photographies originales sur film.

particules dans une chambre à bulles, il est donc nécessaire d'ajuster la grosseur et la densité des bulles en jouant sur les conditions thermodynamiques de la chambre de façon à empêcher la saturation sur toute la longueur des trajectoires.

Remerciement. - Un $\left({ }^{4}\right)$ des auteurs exprime sa (4) E. Hourani. gratitude à M. le Professeur J. Teillac de lui avoir permis de travailler dans l'Institut de Physique Nucléaire qu'il dirige. Il remercie également le C. N. R. S. du Liban qui lui a attribué une bourse de recherches.

\section{BIBLIOGRAPHIE}

[1] Glaser (D. A.) et al., Phys. Rev., 1956, 102, 1653.

[2] Willis (W. J.), Phys. Res., 1957, 108, 1046.

[3] Blinov (G. A.) et al., Sosiet Phys., J. E. T. P., $1957,4,661$.

[4] Kenney (U. P.), Phys. Res., 1960, 119, 432.

[5] Pexrou (G.), Conf. on Instr. for high energy physics, Berkeley, 1960.

[6] Lea (R. M.) et Vitritoe (C.), Res. Scient. Inst., 1962, 33, 243.

[7] Sechi-Zorn (B.) et Zorn (G. T.), Nuovo Cimento, Suppl. 1962, 26, 197.

[8] Fabian (B. N.) et al., Res. Scient. Instr., 1963, 34, $n^{0} 5,484$.

[9] Tridon (J.), J. Physique, 1965, 26, 785.

[10] Ceeccarelli (M.) et Zorn (G. T.), Phil. Mag., $1952,43,356$.

[11] Friesen (S. V.) et Kristiansson (K.), Ark. För Fys., 1952, 4, 505.
Manuscrit reçu le 2 juin 1966.
[12] Waldeskog (B.), Ark. För Fys., 1954, 7, 475.

[13] Johansson (T.), Ark. För Fys., 1958, 13, 129.

[14] Baumann (G.) et al., J. Physique, 1964, 25, Suppl. $n^{0} 11,185$ A.

[15] Yuasa (T.) et al., J. Physique Rad., 1961, 22, Suppl., 175.

[16] Hourani (E.), Thèse $3^{\mathrm{e}}$ cycle, Institut de Physique Nucléaire, Orsay, 1966.

[17] De Vaucouleurs (G.) et al., Manuel de Photographie Scientifique, Édition de la Revue d'Optique, Paris, 1956.

[18] Askar'ian (G. A.), Soviet Phys., J. E. T. P., 1957, $4,761$.

[19] Tenner (A. G.), Nuclear Inst. and Meth., 1963, $22,1$. 